\title{
Écrire (tout) contre : Si près de (Georges) Cixous
}

Writing (Right) Against: Si près by (Georges) Cixous

\section{Sylvain Dournel}

\section{OpenEdition}

Journals

Édition électronique

URL : https://journals.openedition.org/recherchestravaux/2622

DOI : $10.4000 /$ recherchestravaux.2622

ISSN : 1969-6434

Éditeur

UGA Éditions/Université Grenoble Alpes

Édition imprimée

ISBN : 978-2-37747-241-3

ISSN : 0151-1874

Référence électronique

Sylvain Dournel, «Écrire (tout) contre : Si près de (Georges) Cixous », Recherches \& Travaux [En ligne], 97 | 2020, mis en ligne le 12 novembre 2020, consulté le 02 juillet 2021. URL : http://

journals.openedition.org/recherchestravaux/2622 ; DOI : https://doi.org/10.4000/recherchestravaux. 2622

Ce document a été généré automatiquement le 2 juillet 2021.

(C) Recherches \& Travaux 


\section{Écrire (tout) contre : Si près de (Georges) Cixous}

Writing (Right) Against: Si près by (Georges) Cixous

\section{Sylvain Dournel}

\section{1. Écrire avec}

1 «La mort de mon père, je ne l'ai pas crue, j'ai cru plus fort en sens inverse, j'ai fait un bond en arrière, je me suis enfermée dans une réalité autre, instituée par moi » ( $S P$, p. 19) s'entête Cixous à l'orée du texte, inaugurant autour du «je», lecteur et spectateur du Moi, ce parti-pris du "sens inverse", du «à rebours", que Si près va tenter de porter à bout de récit et surtout de discours. Posture intenable où le tout de l'existence, et donc du Livre dans le cas présent, s'arrime à la toute-puissance - si nietzschéenne - du postulat :

J'ai toujours su que je savais qu'il ne reviendrait pas, mais savoir ne pèse rien à côté de vouloir, inventer, maintenir. Ce que je sais n'est rien. C'est ce que je veux et peux qui est la vraie réalité. Je disais : il n'est pas mort. Ce n'était pas moins vrai que le contraire. (SP, p. 19)

2 La mort ne serait alors qu'une vie en creux ou en négatif, niable à volonté et dont le poids n'affecte en rien « la vraie réalité », ce que dément sans surprise l'intégralité de la fiction à venir, du - longuement fantasmé - voyage en Algérie à la rencontre hallucinée avec le père, nous y reviendrons. Plutôt que de s'ancrer durablement dans l'artifice consenti de la négativité, Si près s'érige très tôt en lutte - «j'use un titan par page» (SP, p. 141) avoue l'auteure - contre l'évidement progressif d'une présence promise à l'oubli, encasernée dans un silence et un repos auxquels l'écriture compte bien refuser l'éternel :

Le passant à qui l'on raconterait le secret de cette tombe n'éprouve pas plus de pitié que pour une feuille morte. La mort c'est ça : rien ne reste, ni le souvenir de la vie, ni le souvenir de la souffrance, ni le souvenir de la mort, ni le souvenir de l'oubli. (SP, p. 20) 
Remonter obsessionnellement le cours de cet oubli, à contre-courant du silence des autres et de son propre silence, voilà qui va aimanter jusqu'à leur terme la parole et la voix du récit ; la matrice est en place et Si près, avide d'appel, de présence et de devenir bien plus que de mémoire, peut s'engager sur une voie très proche du questionnement barthésien :

Écrire pour se souvenir? [interroge Barthes] Non pour me souvenir mais pour combattre le déchirement de l'oubli en tant qu'il s'annonce absolu. Le - bientôt " plus aucune trace », nulle part, en personne ${ }^{5}$.

Écrire avec la mort donc, tout contre le défunt plutôt que contre son absence; le parcours du récit s'établit entre la violence définitive d'un constat initial, «il m'a toujours semblé que j'étais née "comme arrachée de sa tombe" " $(S P, p .37)$, et l'apaisement conclusif du don de soi, «lui donn[er] mon être pour qu'il soit " (SP, p. 208). Entre ces deux pôles, le travail de l'écriture aura été de faire coïncider cet être avec celui du défunt, de lui offrir les conditions d'une naissance bien plus que d'une survie ; «Pour moi tout vient de mourir. Je l'ai toujours su' ${ }^{6}$ " affirme ailleurs Cixous, une conviction qui n'a que bien peu à voir avec la conscience de la finitude humaine. Au-delà d'une écriture de l'urgence à vivre ou d'une quelconque écriture-échappatoire, écrire induit un mouvement transgressif, le tissage d'un lien à même de convertir la disparition en trait d'union, à même également de signer un triple acte de naissance : naissance du discours incarné, vocalisé qui s'oppose à la perte, re-naissance du défunt à qui la fiction prête souffle - Derrida parlera de « métempsychose infinie ${ }^{7}$ » au sujet du romanesque cixousien -, fonction ontogénique enfin puisque de cette lutte émerge un être-au-monde transfiguré. Affranchissement de la limite et du seuil imposés disionsnous, l'écriture témoigne ici d'un hybris - ou pour le moins d'une volonté hybristique - que revendique volontiers l'auteur du Détrônement de la mort ${ }^{8}$, elle qui va jusqu'à l'instituer en critère définitoire de la « vraie littérature » :

Je dirais qu'on distingue la vraie littérature de la littérature commerciale par cela: qu'est-ce que cela sauve? Qu'est-ce que cela sert? Je ne parle pas de cause. C'est toujours en rapport avec une menace de la mort de quelque chose de vivant, de fragile, de précieux'.

C'est bien ce creux originel qui sert de plein au récit, cette «menace » qui pour lui fonctionne comme force d'innervation. Le texte y nourrit son «effet Détourretournant» (SP, p. 33), comme l'appelle Cixous, une coexistence ténue des contraires où l'absence fait vivre, l'inhumation exhume et la mort est promue avocate du principe vital. À cette entreprise hors norme, faite d'échappée de soi et de reconstruction d'un existant, le langage n'offre qu'un appui fuyant ; en lieu et place du mot juste, qui révèle, éclaire, convoque la présence en nommant, "[à] chaque pas on s'enfonce, les mots pivotent » (p. 66) et se refusent à colmater l'absence.

6 Sommé de signifier, le nom peine à libérer sa substance de substantif. Commun, il demeure obstinément celui des autres, pré-dit, mal dit ou trop dit, peau morte et dégoût :

Ma peau, cette colle mortelle de mots pilés, mêlés, étalés en poison sur ma vie psychique [...] une colle de mots remâchés en recrachats sur l'être. (SP, p. 102)

7 Propre, le nom se cloisonne dans sa rigidité stérile et condamne tout accès, y compris celui de la terre où retrouver le père : "Qu'est-ce que c'est "Algérie" ? Sans article la chose n'est plus qu'un mot. Un signifiant étrange.» (SP, p. 47) Face aux présumées traîtrises ou apories du langage, la nomination chez Cixous se résout ainsi, dans 
l'indécidable, entre assignations fermes, résolues, ostentatoires du signifiant au signifié et négations de toute possibilité d'affirmer ; puisque « c'est le non-nommé, l'invoqué, le secret, l'adorable, qui est l'essentiel» (SP, p. 209), la signification divorce du signe, ou s'opèrera dans un au-delà de ce signe.

\section{Stratégies d'un faire revenir}

8 Mais puisque le deuil est ici à l'origine de l'énonciation, et qu'il ne peut rester silence, les modalités de ce faire advenir, ou de ce faire revenir, sont plus certainement à chercher du côté des stratégies énonciatives et discursives mises en œuvre. Pour que l'écriture capture, comme un appareil photo scriptural, «le flux infini de l'imprenable » $(S P$, p. 11), elle ne peut que tenter d'épouser cette logique extensive, de la germination et de l'engendrement continus, en un flux qui entrelace intuitivement récit et discours, sous la pulsion d'une « contraction réflexe de l'âme » $(S P$, p. 55). Tissé par l'aléatoire de monologues intérieurs qui juxtaposent, imbriquent ou concatènent les discours, le texte pluralise les sources de sa parole, accueille le tout-venant de la pensée, se fait souple, plastique, organique. Graduellement le récit se déréalise, fusionnant de manière baroque l'existant et l'onirique, le tangible et l'abandon à l'illusion. Nouvelle ère du soupçon et du doute, nouveau mentir-vrai où réalité et fiction se coécrivent; la scène inaugurale du maillot de la mère octogénaire peut sans transition faire place à la lettre à Zohra Drif, au Téléphone imaginaire, au vol onirique pour l'Algérie, aux allégories animalières, à la rencontre enfin... Un flux - lexical, syntaxique, lyrique souvent, épique parfois - qui assemble l'épars d'une vie en un succédané de possibles; " chambre aux porcelaines », " danse ailée de l'idée » écrirait Segalen, où le miracle au détour d'une phrase s'adosse au «bon gros réel ».

9 Manquent encore à ces vertiges discursifs et diégétiques, faits d'élans, de pulsions et d'adresses, les moyens d'un réel franchissement de la distance - spatiale et temporelle - qui sépare énonciatrice et défunt. C'est bien, nous le pressentions plus haut, au-delà du signe que le miracle peut avoir lieu. Et c'est à l'intérieur de ce que l'on pourrait appeler chez Cixous une pensée tropologique, tant le texte progresse par bonds ou à-coups, qu'il va pouvoir être verbalisé. Dans une perspective très générale, le trope, détournement de sens de la littéralité vers la figuralité, organise l'espace linguistique en fonction de celui du sensible, instaurant un lien entre ces deux espaces de référence, ceux d'Hélène et de Georges Cixous en l'occurrence dans Si près. À l'intérieur de ce jeu qu'introduit le trope, dans cette perturbation de la relation étroitement codifiée entre signifié et signifiant, se jouent la communion que le texte cherche à établir ainsi qu'un sens toujours en quête de lui-même, ouvert sur un extérieur du discours, résolument en puissance et dans le cas présent, en souffrance. En d'autres mots, les configurations figuratives mises en œuvre ici font du signifiant un levier, un ouvroir, une arme de jet vers un ailleurs du texte.

10 Trope de condensation qui avère ce «mouvementement» (SP, p. 11) de l'écriture, le mot-valise, « tourné » sur l'axe de la syllabe centrale du signifiant, est celui qui apparaît avec le plus d'évidence dans le texte; un au-delà déjà des possibles de la langue, première étape de ce bond vers le père et l'indispensable Algérie. Lieu focal mais aussi nodal de Si près, il cristallise d'emblée les réseaux connotatifs du texte, entre «algériance » $(S P, 21)$, «exalgériance» et "algirritation» (SP, p. 71) que le «pourquoiement» maternel «entrebroussaille» à intervalles réguliers. Par le 
syntagme créé, il s'agit de s'approcher, de s'approprier, de faire sienne la terre tombale, de la saisir pour mieux l'investir.

11 Deuxième étape de ce processus, la métonymie - et Cixous avoue dans le métatexte procéder "par métonymies, par intuitions, par détours» ( $P$, p. 56) - autorise davantage encore cette saisie en offrant, à l'imaginaire comme au texte qui en constitue le support, une image à étreindre. L'opération de substitution qu'induit la métonymie va charger « Algérie » d'une signifiance seconde, synthétiser la plus-value cognitive que le texte a patiemment construite et autour de laquelle il peut générer d'autres références. Le méronyme "Alger »- la partie du tout «Algérie »- peut exercer son pouvoir de contamination ou d'irradiation, jusqu'au vertige :

Le mot Alger n'était venu que par métonymie, j'étais prête à le jurer. Je n'avais pas voulu dire que j'irais peut-être voir mon père, ou bien la tombe de mon père, c'està-dire mon père par métonymie, ou bien la métonymie de mon père, les substitutions les plus incroyables [...]. (SP, p. 51)

Lentement, le trope infuse le texte, s'abolit dans sa fonction figurative, à l'exemple du cyprès, celui des années lycée puis des allées du cimetière d'Alger, qui par contiguïté référentielle, connexion ou inclusion, est aussi l'Algérie, l'adolescence, le père. Même la parole de ce dernier semble avaliser cette manière de penser l'existant, et donc de l'écrire :

J'écris un texte tout traversé par toi, dis-je, même s'il semble l'être par quelqu'un d'autre que toi. - J'ai l'habitude. - De quoi ? - D'être remplacé. (SP, p. 208)

Fondu dans le discours, sans rupture isotopique, le mouvement se crée autour du cyprès, déplace les sèmes, dilate sa référence, le texte s'établissant dans le flux des appels connotatifs et des cotopies ${ }^{10}$ qu'il a lui-même constitués. Dès lors et par extensions successives, il s'architecture autour d'une ample chaîne métonymique. Le récit se ramifie, l'enjeu sémantique de la lecture se double ; dire sans dire, ou dire sans nommer, l'ellipse métonymique ne fournit aucune clef, se refuse à toute unilatéralité mais ouvre d'autres possibles.

Promu symbole, ce même cyprès peut alors déployer toutes les virtualités du texte. Plus encore que l'allégorie, car plus intuitif et dénué de valeurs figurées stables, il cultive en tant que symbole non constant son autonomie, n'impose aucune signification en surface du texte tant il est structuré par les caractères qui lui sont - et demeureront propres. C'est grâce à cette opacité prometteuse que le texte démultiplie sa capacité à signifier, à faire signe; c'est là que Siprès trouve son titre, son acmé et son - provisoire - épilogue. Le cyprès fonctionne à la fois comme pierre d'attente, pierre angulaire et pierre de touche du récit. Il est d'emblée "mon facteur mystique » dont "la flèche file vers mon père" ( $S P, \mathrm{p} .86)$. Anthropomorphe il devient, dès son apparition et avec la force de l'évidence, corps du père: «Mince, élancé, flexible, emporté dans une exaltation sobre, je vois que c'est le corps de mon père.» (Ibid.) Une fois introduit, le symbole "cyprès " peut jouer de sa multivalence. Il est aussi un symbole constant, celui de l'arbre tel que l'ont célébré Hugo, Claudel ou Giono: une racine-passé et un feuillage-avenir, un vivant trait d'union entre terre et ciel, une sagesse interposée entre hommes et dieux, un père enfin, autour duquel se tiennent les conciliabules $^{11}$. Importé dans le diégétique, dans le discursif, le cyprès devient symbole iconique, celui d'une impossible relation, d'un improbable commerce auquel il offre cependant une modalité d'existence: la rencontre hallucinée pourra avoir lieu, le dialogue d'outre-tombe s'engager et la narratrice enfin tenir "l'infini par un bout», poser « [s]a main sur son pied» $(S P$, p. 87$)$ : «Je n'ai jamais été si près.» $(S P$, p. 137) 
L'horizon d'attente fixé par le titre se comble, mais le symbole s'épaissit encore. L'homophonie entre le substantif et la locution adverbiale est créatrice d'un nom verbal, prédicatif : le cyprès permet d'être si près. Et le cyprès de se gonfler de sens, jusqu'à éclater: "Et le bruit de la source coupée. Les deux langues de mon père, coupées. L'arabe, l'hébreu. - abe. - breu. Arbreu. Comment recoudre une voix ?» (SP, p. 138)

\section{3. « L'outre-vie »}

Puisque l'écriture est trace, et trace vivante, vibrante, organique, cette voix s'avère une instance essentielle du récit; en tant que signature d'un corps énonciateur, elle parachève son inscription au texte, dans l'intime, jusqu'à ce qu'elle se dote d'une modulation, d'une tessiture qui fasse sens, dans un infra - ou ici supra - sémantisme. Le sonore se fait alors intensification du voir, mise en tension de la présence, une gestuelle locutoire qui fait ici mouvement vers le défunt. La voix des premières pages - «Voix étonnée, ascendante, venue du fond des temps, atteignant les aigus de l'incrédulité. Pause. Voix redescend la pente. » (SP, p. 47) - monte ainsi graduellement en puissance, jusqu'à se démultiplier en un ensemble de choreutes, dirigé par un «Je » coryphée, pour crier le nom du père, «Cixous Georges » (SP, p. 200). La rencontre peut enfin avoir lieu, totalement, et sur un mode délibérément synesthésique :

Et pendant que je vois j'écoute. Ce qui se passe est simultanément chant. D'une certaine manière un opéra m'habite. Ce que ma main fait couler sur le papier c'est ce que je vois-entends, mes yeux écoutent, ma chair scrute ${ }^{12}$.

Avant que le père, en guise d'écho, ne fasse lui aussi mouvement vers elle, la narratrice n'a plus qu'à laisser place à cet être originel, primitif mais intermittent, né en elle pour mener à bout ce « miracle / Auquel personne en ce monde ne peut croire» $(S P$, p. 210) :

Celui qui mène c'est ce corps primitif, l'être du contact, celui qui prend le commandement après le couvre-feu, qui fait les ascensions des montagnes devant lesquelles je suis paralysée, qui a un pacte immémorial avec les lignes de la terre, qui nage jusqu'aux villes éloignées comme s'il était conduit dans l'inconnu par les voix secrètes des générations, il sent que l'on va arriver devant le Cimetière. ( $S P$, p. 146)

Redoublée par l'accélération subite de la vitesse narrative, la dramatisation de l'acmé $\mathrm{du}$ récit va d'ailleurs thématiser la concomitance de ces présences, vocale et ontologique. "Notre téléphone surnaturel est maintenant branché, toute la montagne frémit de ton murmure » $(S P$, p. 202) s'enthousiasme la narratrice. Mais à son « Où estu? " ne répond qu'un allusif «Si près », bientôt suivi d'un «es-tu? " aussi total que définitif. Ni retrouvailles, ni célébration lyrique, ni message, ni réponse dans cette prosopopée minimaliste; la très courte séquence dialogique, où enfin le père cesse un instant d'être un dit pour s'actualiser d'un dire, n'aura donné lieu qu'à un questionnement abrupt, un renvoi vers un extérieur du discours, nécessairement déceptif. Les lignes tensives de l'écriture, qui depuis l'incipit s'étaient étoilées autour de cet instant, peuvent à nouveau se retourner vers l'intériorité de la narratrice.

Pour autant la quête n'aura pas été vaine. La théâtralisation graduelle du récit l'a contraint à trouver son lieu, lieu qui a vectorisé ses forces depuis les premières lignes ; le deuil ne peut rester sans contact avec sa source. Outre la distance, la mère ne s'est pas révélée un adjuvant de choix; le Cimetière, "pourtant devenu un personnage puissant et redoutable de cette scène » assure la narratrice, "n'a pas besoin de toi » 
$(S P$, p. 53-54) tranche sa mère, qui n'est pas du genre à parler à un cyprès et demeure résolument et obstinément rangée du côté des forces vitales :

Ma mère est jalouse du Cimetière. Elle lui prête les sentiments qu'elle voudrait qu'il ait. Elle désire obscurément que le Cimetière me rejette, ce serait un châtiment mérité pour celle qui prend le Cimetière pour son père, au lieu d'aller faire un tour au supermarché avec sa mère. Mais peut-être a-t-elle seulement voulu me dire : il est trop tôt pour mourir. On n'a pas besoin de ça. (SP, p. 54)

Il n'en fallait sans doute pas plus pour que le récit s'érige, selon le néologisme de Cixous, en " recimetière ${ }^{13}$ ", une actualisation de celui d'Alger, une mise au présent du souvenir où la tombe ${ }^{14}$, au terme du parcours, s'élève en ultime signifiant. Évidemment pour la mère, toujours pragmatique, dans la tombe «rien du tout [...] Au bout de quelque temps. » (SP, p. 80) Mais pour la narratrice, elle est l'objet d'un questionnement fécond - «Se tient-elle hors texte? Me tient-elle hors texte?» (SP, p. 199) - un questionnement qui aiguise ses velléités de départ. Plus encore c'est la bivalence de la pierre tombale qui cristallise ses incertitudes : elle est à la fois "tombe sans adresse", isolée dans l'absence anonyme, et "signature» (SP, p. 199), trace tangible d'une présence, une stèle levée contre le vide et à laquelle le texte va prêter vie : «je sentais comme elle était vivante» ( $S P$, p. 205) s'exclame la narratrice, en étreignant son "visage de granit» $(S P$, p. 210). La découverte de ce granit-peau, devenu le père, est sans doute la plus pérenne du récit, et entremêle "je ", texte et disparu, devenus consubstantiels: "Avec ma voix j'ai creusé en moi la pierre» (SP, p. 207); une immersion et une émersion simultanées, à l'interface de l'écrire et de l'existant.

Dans cette perspective édificatrice, le temps de Si près ne saurait bien sûr rester le passé du remémoré ou du remémorable. L'écriture pour Cixous est par essence élaboration; même et surtout si elle prend la mort pour objet, elle demeure tout entière orientée vers la construction d'un sujet, auquel « le Récit », hypostasié par la majuscule, accorde le don du temps :

Moi, le Récit, (écrit Cixous dans Déluge ${ }^{15}$ ) je vous donne le temps, je ne vous l'ôte pas, j'ouvre par mes paroles le livre de la mort; et à la Mort aussi je donne le temps vivant, ce temps qu'elle n'a jamais [...]. Nous ne sommes pas coupables, nous sommes à l'avance, nous sommes sans espoir, jamais deuil n'aura eu si grand et pur rayonnement. La personne adorée resplendit devant nous, vivante nous l'aimons comme jamais et pour jamais plus, jamais créature n'aura été tant adorée d'avance et pour cent années, le voilà mort tout vivant devant nous la voilà déjà morte et pourtant pas du tout, demain et aujourd'hui entrechoquent leurs heures d'or et d'argent.

21 L'écriture est par conséquent ce qui permet à nouveau l'écoulement du temps. Si près a d'ailleurs, dès ses premières pages, simplement - et ostensiblement - tourné le dos à la temporalité chronologique et s'est soustrait au flux linéaire du chronos pour s'accorder au temps de l'événement, au temps humain de Benveniste, celui de $l^{\prime}$ « expérience humaine ${ }^{16}$ ", défini et ordonné à l'intérieur du discours. Cette conception du temporel, ou de l'extra-temporel, qui fait du récit « un prétexte à détricoter le temps» (SP, p. 74), promeut logiquement les tiroirs verbaux longs, qui éternisent l'instant, une syntaxe du flux qui déponctue volontiers le texte, refusant de césurer cet instant, à l'exemple également des pages finales du récit, celles de l'après-rencontre, non numérotées, sans ordre dans un temps sans balises. Et puisque le deuil n'aura pas de fin, le récit ne peut l'évoquer que dans son inaccompli, le discours dans son informulé : «Je vois tout de l'autre côté, comme dans l'outre-vie, outre-mémoire. » (SP, p. 205) 
Si près, à l'exemple de l'œuvre cixousienne, se donne ainsi à lire comme une affirmation dont le paradoxe fait l'épaisseur, entre la lyrique isolée d'un «je » et l'émergence d'un universel : «Je suis inconsolable. C'est une consolation sans limites. Il y a dans l'âme un infini, c'est le chagrin. Le chagrin est immortellement jeune.» (SP, p. 212) L'échec de l'écrire contre, stérile et statique, s'efface au profit d'un écrire tout contre et même d'un écrire vers, une poétique singulière où la mort n'est qu'une présence absentée, où le dit du deuil peut servir de palimpseste au dire du deuil, inachevable, où le récit n'est pas - ou pas seulement - narration, mais prospection. De la quête à la voix d'outretombe, l'écriture, plus que jamais inchoative, exerce sa prérogative première : passer outre.

\section{NOTES}

1. J.-M. Maulpoix, Du lyrisme, Paris, Corti, 2000, coll. « En lisant en écrivant », p. 424.

2. H. Cixous, Si près, Paris, Galilée, coll. «Lignes fictives», 2007, p. 12. Les références à ce texte seront données entre parenthèses $(S P)$.

3. Voir H. Cixous, OR, les lettres de mon père, Paris, Des femmes, 1997 ; Ève s'évade : la ruine et la vie, Paris, Galilée, coll. «Lignes fictives", 2009; Homère est morte..., Paris, Galilée, coll. «Lignes fictives ", 2014.

4. H. Cixous, Si près, Paris, Galilée, coll. « Lignes fictives », 2007.

5. R. Barthes, Journal de deuil, Paris, Seuil, 2009, p. 79.

6. R. de Ceccaty, « Hélène Cixous : les secrets d'une résistance », Le Monde, 14 juin 2003.

7. J. Derrida, «H.C. pour la vie, c'est-à-dire... », dans Hélène Cixous, croisée d'une œuvre, M. CalleGruber (dir.), Paris, Galilée, coll. « La philosophie en effet », 2000, p. 32.

8. H. Cixous, Le Détrônement de la mort. Journal du Chapitre Los, Paris, Galilée, coll. « Lignes fictives », 2014.

9. Séminaire d'Hélène Cixous, 1985-1986. Centre d'études féminines à l'Université Paris VIII, Saint-Denis.

10. La cotopie est la relation sémique qui unit sens propre et figuré. Voir M. Bonhomme, Linguistique de la métonymie, Berne, Peter Lang, 1987.

11. P. Claudel, "Le Banyan», dans Connaissance de l'Est, Cuvre poétique, Paris, Gallimard, coll. « Bibliothèque de la Pléiade », 1957.

12. H. Cixous, «La Venue à l'écriture », Entre l'écriture [1976], Paris, Des femmes, 1989, p. 64.

13. H. Cixous, Le Jour où je n'étais pas là, Paris, Galilée, coll. «Lignes fictives », 2000, p. 167.

14. Cette tombe deviendra l'héroïne éponyme d'un autre roman d'Hélène Cixous (Tombe, Paris, Seuil, 2008).

15. H. Cixous, Déluge, Paris, Des femmes, 1992, p. 96-97.

16. É. Benveniste, «Le langage et l'expérience humaine ", Problèmes de linguistique générale, t. 2, Paris, Gallimard, 1974, p. 67-78. 


\section{RÉSUMÉS}

Même si elle tend à diluer tout référentiel trop ostensiblement autobiographique, l'œuvre d'Hélène Cixous s'origine - de manière explicite, continuelle et assumée - dans le vu, lu et vécu de celle qui très tôt a vu son existence ponctuée par les disparitions : du père, Georges, en 1948 alors qu'elle est âgée de dix ans, plus tard du fils, de Gregor l'amant, de Derrida l'ami et plus récemment de la mère, Ève. Logiquement, le deuil constitue l'épicentre - toujours actif et à vif des récits mais pour autant le texte cixousien se refuse à tout attendu, tout pré-écrit; ni déploration ni pathos, peu de lyrisme effusif, "plaintif et de pompe à larmes ${ }^{1}$ » fustigerait Maulpoix. En lieu et place de cette topique - et puisque le deuil suppose, appelle un "travail ", au sens parfois étymologique du terme - la «machine à écrire intérieure ${ }^{2}$ » de Cixous se fait funambule, en équilibre sur l'abîme de l'absence et tendue vers la restitution d'une présence. Certes l'écriture, comme souvent, avère chez l'auteure d'OR, les lettres de mon père, d'Ève s'évade ou d'Homère est morte... ${ }^{3}$, ses vertus thérapeutiques et cathartiques, mais ici - et tout particulièrement dans Si près ${ }^{4}$ - c'est bien d'une quête dont il s'agit. Quête d'une modalité de résistance à la limite, à tout seuil définitif et ultime ; écrire contre la perte et l'inéluctable de son aval. Quête également d'une voie possible, d'une modalité du faire revenir symptomatique d'un rapport singulier au réel, donc à l'écriture, au langage, à l'espace, au temps ; conquête enfin, à force de réminiscence, de désir, d'atermoiement, de résolution, de refus, de procrastination, de folie et d'amour, d'un écho, d'une voix, d'une réponse.

Even though it aims at watering down any reference too obviously autobiographical, Hélène Cixous' work roots-explicitly, continuously and assumedly-into the seen, read and lived of a woman whose existence was rhythmed by disappearances since early childhood: her father, Georges, in 1948 when she was ten years old, then her son, her lover Gregor, her friend Derrida and more recently her mother, Eve. Mourning constitutes the logical heart-still persistent and raw-of the stories, however the Cixousian text refuses to be pre-written or pre-expected; neither lament nor pathos, few effusive lyricism, or "plaintive and teardrop pump", would say Maulpoix. Instead -and since mourning presupposes, calls for a "work", in its etymological senseCixous' "inner typewriter" becomes a tightrope walker, walking above the abyss of loss and reaching for the restitution of a presence. Of course, writing, as often, inspires the author of $O R$, les lettres de mon père, of Ève s'évade or Homère est morte... some therapeutic and cathartic virtues, but here-and especially in Si Près-it is more about a quest. A quest for a how to resist the limit or any definitive and ultimate threshold; how to write against the loss and the inevitability of what comes before. It's also a quest for a possible path, for a way of a symptomatic return of a singular relationship to the real, therefore to writing, language, space and time; the conquest, finally, with reminiscence, desire, lamentation, resolution, refusal, procrastination, madness and love, of an echo, a voice, an answer.

\section{AUTEUR}

\section{SYLVAIN DOURNEL}

Université de Lille 